\title{
Dinámica de oviposición de Aedes (Stegomyia) aegypti (Diptera: Culicidae), estado gonadotrófico y coexistencia con otros culícidos en el área Metropolitana de Bucaramanga, Colombia
}

\author{
Oviposition dynamics of Aedes (Stegomyia) aegypti (Diptera: \\ Culicidae), gonadotrophic state and coexistence with other \\ Culicidae in the metropolitan area of Bucaramanga
}

\author{
Nicolás Ruiz ; Gustavo-Adolfo Rincón ${ }^{1}$; Héctor-Julio Parra ${ }^{1}$; Jonny-Edward Duque ${ }^{1}$
}

Forma de citar: Ruiz N, Rincon GA, Parra HJ, Duque JE. Dinámica de oviposición de Aedes (Stegomyia) aegypti (Diptera: Culicidae), estado gonadotrófico y coexistencia con otros culícidos en el área Metropolitana de Bucaramanga, Colombia. Rev Univ Ind Santander Salud. 2018; 50(4): 308-319. doi: http://dx.doi.org/10.18273/revsal.v50n4-2018004 (c) (1)

\section{Resumen}

Introducción: Las zonas urbanas tropicales favorecen la presencia de Aedes aegypti en el continente americano. Otros culícidos pueden compartir la misma área de distribución con este mosquito, lo cual justifica que se deban monitorear. Objetivo: Conocer la dinámica de oviposición de A. aegypti, evaluar su estado gonadotrófico y determinar taxonómicamente la presencia de otros culícidos en el área metropolitana de Bucaramanga. Metodología: Para este propósito fueron instaladas trampas de oviposición $(\mathrm{N}=960)$ cebadas con infusión de heno $(10 \%)$ durante un año en cuatro localidades (Norte, Girón, Piedecuesta y Floridablanca). Las ovitrampas permanecieron instaladas durante cinco días en intradomicilio y peridomicilio, paralelamente se colectaron larvas en plantas como bromelias y depósitos de agua en cada lugar de colecta. En el mes de mayo de 2014 fueron capturadas con un aspirador eléctrico y trampas StegTrap hembras de A. aegypti y Cx. quinquefasciatus a las cuales se les estimó su estado gonadotrófico. Datos ambientales como precipitación y temperatura fueron correlacionados con índices de oviposición. Resultados: Se obtuvieron 25.254 huevos de A. aegypti (Girón 39,1\%, Bucaramanga 36,8\% Floridablanca 15,2\% y Piedecuesta $8,9 \%)$ en general los índices de oviposición fueron mayores en peridomicilio con correlaciones positivas (> 0,5 ) entre los índices NOP, NH e IPO en peridomicilio y IPO y IDH en intradomicilio con la precipitación y la humedad. En cuanto al estado gonotrófico se determinó que las hembras de A. aegypti presentaron hasta dos ciclos gonadotróficos en las áreas examinadas. En las colectas activas en peridomicilio se encontró, Culex spissipes, Culex quinquefasciatus, Limatus durhamii, Toxorhynchites spp y Wyeomyia davisi. Conclusión: A. aegypti fue presente en todas localidades y se reportan otros culícidos potenciales vectores en el área metropolitana.

Palabras clave: Oviposición; Monitoreo; Vigilancia poblacional; Control de mosquitos.

1. Universidad Industrial de Santander. Bucaramanga, Colombia.

Correspondencia: Jonny Edward Duque. Dirección: Sede Universidad Industrial de Santander, Parque Tecnológico y de Investigaciones Guatiguará. Km 2 Vía El Refugio. Piedecuesta. Telefono: 5776344000 Ext 3503. Correo electrónico: jonedulu@uis.edu.co 


\begin{abstract}
Introduction: Tropical urban areas favor the presence of Aedes aegypti in the American continent. Other mosquitoes can share the same geographic distribution with this mosquito, which justifies that they must be monitored. Objective: It is to know the dynamics of oviposition of $A$. aegypti, evaluate its gonadotrophic state and determine taxonomically the presence of other culicidae in the metropolitan area of Bucaramanga. Methodology: Oviposition traps $(\mathrm{N}=960)$ were baited with hay infusion $(10 \%)$ for one year in four locations (Norte, Girón, Piedecuesta and Floridablanca). The ovitraps were kept for five days indoors and outdoors, while larvae were collected in plants such as bromeliads and water reservoirs from each collecting site. In the month of May 2014, were captured with an electric aspirator and traps to catch females of $A$. aegypti and Culex quinquefasciatus to which were estimated their gonadotrophic state. Environmental data such as precipitation and temperature were correlated with the collected eggs and their oviposition indices. Results: 25,254 eggs of A. aegypti were obtained (39.1\% Girón, 36.8\% Bucaramanga, 15.2\% Floridablanca and 8.9\% Piedecuesta). In general, oviposition rates were higher outdoors. As for the gonotrophic state, it was detected that females of $A$. aegypti can have for over two gonadotrophic cycles. In the active catch outdoors were found Culex spp., Culex quinquefasciatus, Limatus durhamii, Toxorhynchites spp and Wyeomyia davisi. Conclusion: A. aegypti was present in all localities and other mosquitoes are reported in the metropolitan area.
\end{abstract}

Keywords: Oviposition; Monitoring; Population Surveillance; Mosquito control.

\section{Introducción}

Los culícidos (familia Culicidae) son insectos hematófagos vectores de patógenos que causan enfermedades al hombre y animales domésticos. Se estima que esta familia de dípteros puede tener más 3.553 especies, de las cuales en Colombia hay registradas $324^{1}$. Las enfermedades que transmiten estos zancudos están relacionadas estrictamente con la biología, distribución espacial, disponibilidad del huésped y el agente etiológico que hospeden. De acuerdo con esto, algunas enfermedades como ejemplo la Malaria, Filariosis y Encefalitis son mayormente circulantes en áreas rurales y selváticas. A diferencia del Dengue, Chikungunya y Zika donde la transmisión se da ampliamente en áreas urbanas debido a la gran cantidad de personas susceptibles y condiciones adecuadas para el desarrollo de su principal vector $A$. aegypti ${ }^{2-6}$.

Uno de los aspectos clave para ejercer estrategias de disminución de los casos de Dengue, Chikungunya y Zika es mantener el vector en niveles poblacionales bajos, pero para esto es necesario hacer vigilancia entomológica que revele la distribución espacial, periodos de mayor abundancia y lugares de cría de A. aegypti. Un punto a favor para la reducción de los casos de estas enfermedades es que tienen el mismo vector, por lo cual, las actividades de control a primera instancia son las mismas.

En Colombia, como en otras regiones tropicales, los zancudos tienen todas las condiciones necesarias para proliferar y pocas expectativas en su eliminación ${ }^{5,6}$.
Para el caso específico de $A$. aegypti, en esta región del país, se mantiene presente este zancudo durante todo el año. Lo cual es una consecuencia de los problemas de saneamiento ambiental y de falta de políticas de salud ejecutadas que no logran impactar en la reducción de los criaderos de este insecto, lo cual se refleja en los brotes epidémicos de Dengue, Chikungunya y Zica ${ }^{3,7,8}$.

En las enfermedades transmitidas por culícidos deben estar presente el vector, agente etiológico y el hombre 9 . Para el caso del Dengue, una reducción en el número de casos solo se podría dar si existieran las vacunas para eliminar el virus e impedir su transmisión. De acuerdo con esto, existen expectativas sobre posibles vacunas, pero los expertos indican que aun así, el manejo de esta enfermedad debe ser integrado con diferentes estrategias, donde la más eficaz sigue siendo la eliminación de las formas larvarias y adultas del zancudo ${ }^{10}$. Sumado a esto, los hábitos alimenticios de las hembras de $A$. aegypti incrementan las probabilidades de aumentar los casos de estas enfermedades a causa que esta especie necesita alimentarse de múltiples hospederos para completar el proceso vitelogénico de sus huevos ${ }^{11,12}$.

Para el caso específico de la Ciudad de Bucaramanga y su área metropolitana, se ha evidenciado que el Dengue presenta un comportamiento endémico debido a que frecuentemente se detectan casos autóctonos de esta enfermedad ${ }^{13,14}$. Una preocupación que surge es si este fenómeno de endemismo también ocurra para Chikungunya y Zika, por esta razón es necesario conocer más sobre la distribución y fluctuación 
temporal de $A$. aegypti en esta área del país, ya que en la literatura regional existen pocos estudios sobre este vector y no se conoce de literatura científica de otros culícidos presenten en esta zona metropolitana de Bucaramanga. De acuerdo con estas premisas, este trabajo tuvo como objetivos conocer la dinámica de oviposición de A. aegypti en un gradiente de tiempo, evaluar el estado gonadotrófico de hembras adultas colectadas en campo y determinar la presencia de otros culícidos en el área metropolitana de Bucaramanga.

\section{Materiales y métodos}

\section{Lugares de monitoreo}

La selección de las áreas donde se realizaron las colectas fue determinada de acuerdo con el historial de casos de dengue reportados en el área metropolitana de Bucaramanga y por sugerencia de funcionarios de la Secretarias de Salud de Santander (datos no publicados). Luego de que se confirmó que varias localidades presentaban registros de Dengue se procedió a socializar el proyecto con los líderes las juntas de los barrios Hacienda San Miguel (Piedecuesta), Alicante (Girón), Lagos 2 (Floridablanca) y la Comuna Norte el barrio Divino Niño (Bucaramanga), del área metropolitana de Bucaramanga.

\section{Sistemas de muestreo}

Una vez los moradores de las viviendas estuvieron enterados del proyecto se solicitó que mínimo diez jefes de familia se postularan como voluntarios para la instalación de los dispositivos de colecta de huevos y captura de adultos (ovitrampas y trampa StegTrap), de acuerdo con esto se seleccionaron diez viviendas por barrio para un total de 40 viviendas, en las cuales se entregó un consentimiento informado código 7083 aval comité de ética (CIENCI) donde explicaba el proyecto, una vez firmado se procedió a realizar las colectas.

Para el desarrollo de este estudio se efectuaron colectas activas y pasivas de estados inmaduros y adultos de culícidos presentes en el área metropolitana de Bucaramanga en los municipios de Piedecuesta, Floridablanca, Girón y Bucaramanga, para ésta última en la comuna norte (Figura 1). El muestreo pasivo fue orientado para la recolección de huevos de $A$. aegypti por medio de ovitrampas ${ }^{15,16}$, las cuales son un recipiente plástico de color negro con capacidad de $1.000 \mathrm{~mL}$ y un baja lenguas usado como substrato para ovoposición, que está sujeto al frasco con un gancho sujetapapeles mariposa \# 2. Las ovitrampas fueron cebadas con $400 \mathrm{~mL}$ de infusión de heno, obtenida por fermentación de pasto "estrella africana" Cynodon plectostachyus (Poales: Poaceae) preparado en una relación de 29,19 gr en $7 \mathrm{~L}$ de agua mineralizada, durante siete días y después llevada al $10 \%{ }^{16}$.

Las colectas activas fueron realizadas manualmente con pipetas "Pasteur" y cucharones para tomar las larvas y pupas criadas que se detectaron en los contenedores de los lugares de muestreo. Se utilizó también un aspirador eléctrico modelo" High Power Vacuum" portable con voltaje $12 \mathrm{v}$, dimensiones; 29 $\mathrm{cm} \times 10 \mathrm{~cm} \times 12,5 \mathrm{~cm}$ para succionar los mosquitos adultos presentes en las áreas de estudio.

\section{Instalación de las ovitrampas y realización de las colectas activas}

Los muestreos se iniciaron en el mes de octubre de 2013 y finalizaron en el mes de octubre de 2014. En las casas seleccionadas para el estudio se colocó una ovitrampa en el intra-domicilio y otra en el peridomicilio, dado que el muestreo se realizó en zonas urbanas del área metropolitana de Bucaramanga se establece el peri-domicilio como las zonas externas al alrededor de la vivienda. Los dispositivos instalados guardaron una distancia entre viviendas que nunca fue menor a $200 \mathrm{~m}$, las trampas se dejaron expuestas durante un periodo de cinco días por cada mes de muestreo para estimar los índices de oviposición ${ }^{17-21}$.

Luego las ovitrampas fueron recolectadas teniendo la precaución de filtrar los huevos que se desprenden de las paletas en un papel filtro, así, los huevos fueron contados y llevados al laboratorio de entomología del Centro de Investigaciones de Enfermedades Tropicales (CINTROP) de la Universidad industrial de Santander (UIS). El material colectado se dejó secar entre siete y diez días con el objetivo de permitir la maduración de los embriones presentes en lo huevos, para posteriormente estimular la eclosión. Las larvas obtenidas se alimentaron y se dejaron crecer por cinco días para ser trasladadas a vasos plásticos, dispuestos en contenedores con malla para facilitar su manipulación, alimentación y permitir que los adultos emergieran sin perder escamas y cerdas, para posteriormente ser identificados taxonómicamente.

\section{Índices de oviposición}

En las trampas instaladas mensualmente fueron calculados los índices de positividad de las ovitrampas $(\mathrm{IPO})=(\mathrm{NOP} / \mathrm{NOE}) * 100$, donde NOP es el número de ovitrampas positivas y NOE es el número de trampas examinadas, y el índice de 
densidad de huevos $($ IDH $)=\mathrm{NH} / \mathrm{NOP}$, donde el $\mathrm{NH}$ corresponde al número de huevos ${ }^{19,20}$.

Paralelamente durante la instalación de las ovitrampas se realizaron muestreos activos en bromelias, principalmente de los géneros Guzmania sp (Piedecuesta), Tillamdsia sp (Girón y Bucaramanga) y Neoregelia sp (Piedecuesta), en rutáceas género Dracaena sp (Piedecuesta) y en contenedores de agua residuales de lluvia. Tanto huevos como larvas colectadas fueron llevadas al laboratorio y se mantuvieron hasta la emergencia de los adultos, los ejemplares fueron sacrificados, montados $\mathrm{y}$ determinados taxonómicamente.
La identificación taxonómica de los mosquitos colectados se realizó por comparación de características morfológicas de acuerdo a claves taxonómicas ${ }^{21-23}$. Algunas determinaciones taxonómicas se confirmaron comparando especímenes con la colección entomológica del Laboratorio de Entomología del Instituto Nacional de Salud en Bogotá. Colombia. Los individuos se identificaron hasta especie, sin embargo, los individuos restantes que no coincidieron con distribución espacial con los reportes de especies de culcidae de Rozo-Lopez y Mengual ${ }^{1}$ para Colombia se denominaron "spp".

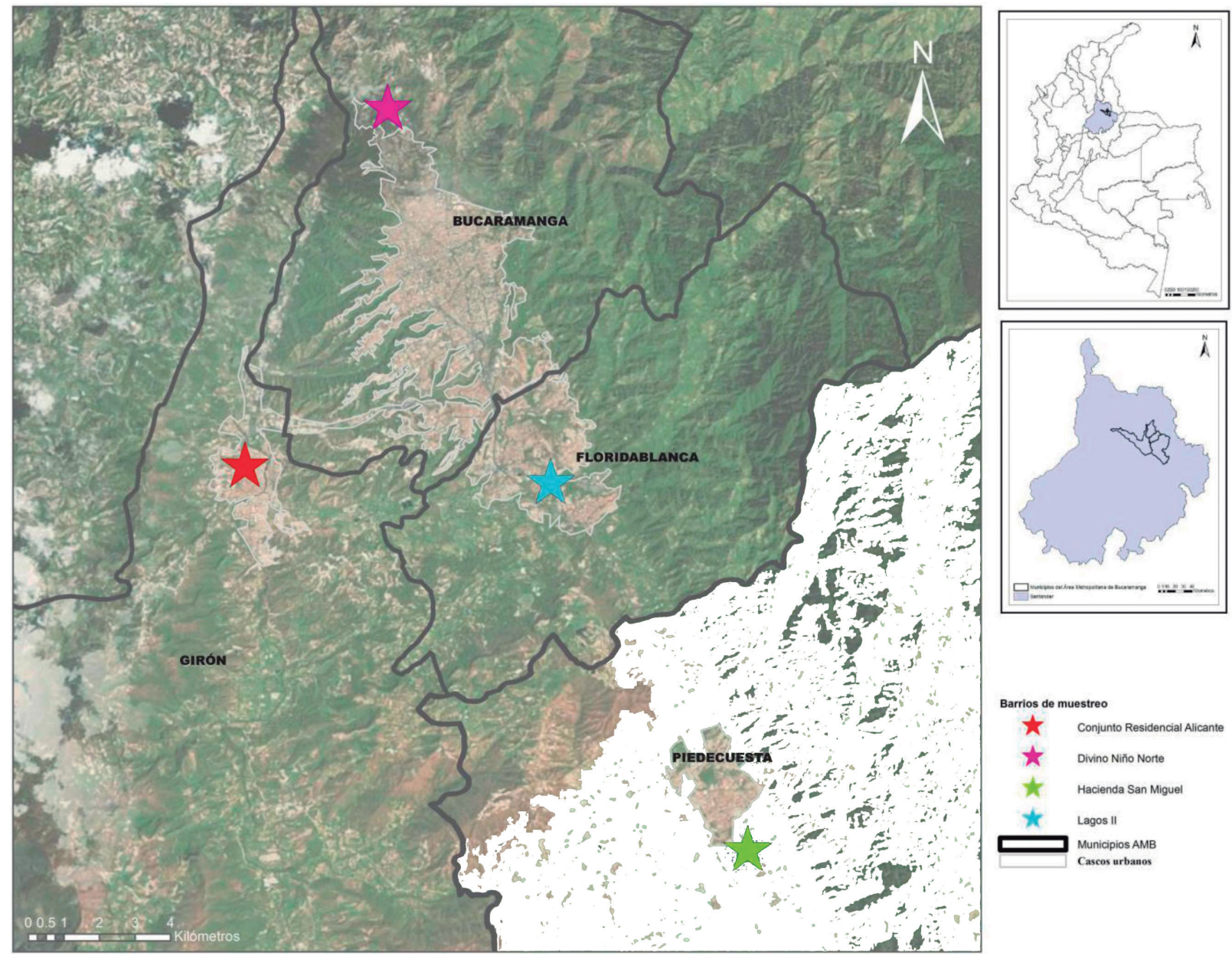

Figura 1. Lugares de monitoreo

\section{Estado gonadotrófico}

En el octavo mes de muestreo correspondiente a mayo y junio se realizó una colecta utilizando trampas para hembras grávidas StegTrap ${ }^{24}$ cebadas con agua instaladas por cinco días para capturar vivos los especímenes para las pruebas, además de este método se uso un aspirador eléctrico accionado durante $15 \mathrm{~min}$ para captura de los adultos presentes en las viviendas (peridomicilio-intradomicilio), paralelo al muestreo de las trampas (ovitrampas), a este material se le determinó el número de ciclos gonadotróficos ${ }^{25-30}$. 


\section{Análisis estadístico}

Todos los datos fueron sometidos a las pruebas de normalidad y homocedasticidad (Kolmogorov Smirnov y Levene). Cada uno de los muestreos donde se hicieron las colectas y se estimaron los índices de oviposición se analizaron con la prueba T-Student para muestras independientes donde se comparó si existían diferencias estadísticas entre áreas intra y peridomicilio. También se hizo un análisis de correlación de Pearson para determinar la relación entre los índices de oviposición y las variables precipitación y temperatura las cuales fueron tomadas del IDEAM para cada localidad. La significancia estadística se consideró con valores de $\mathrm{p} \leq 0,05$, en todos los análisis se utilizó el Software Statistica V11.

\section{Resultados}

Durante el estudio se detectó $A$. aegypti en todos los lugares de muestreo del área metropolitana de Bucaramanga. Se recolectaron 25.254 huevos que corresponden a 39, $1 \%$ en Girón, 36,8\% en Bucaramanga, $15,2 \%$ Floridablanca y $8,9 \%$ en Piedecuesta. Se observó también el número de ovitrampas y los índices de oviposición en general, en el peridomicilio fueron mayor que en el intradomicilio siendo esto estadísticamente significativo para NOE e IPO. Sin embargo, los índices no permitieron detectar un patrón de comportamiento que indiquen los meses de mayor y menor porcentaje de ovoposición (Tabla 1).

Tabla 1. Número de ovitrampas e Índices oviposición obtendidos en el área metropolitana de Bucaramanga.

\begin{tabular}{cccccccccccc}
\hline & \multicolumn{2}{c}{ NOP } & \multicolumn{2}{c}{ NOE } & \multicolumn{2}{c}{ NH } & \multicolumn{2}{c}{ IPO } & \multicolumn{2}{c}{ IDH } \\
\hline Mes de colecta & Intra & Peri & Intra & Peri & Intra & Peri & Intra & Peri & Intra & Peri \\
\hline Octubre 2013 & 13 & 20 & 37 & 37 & 260 & 545 & 35 & 54 & 20 & 27 \\
Noviembre 2013 & 13 & 18 & 40 & 40 & 619 & 1030 & 33 & 45 & 48 & 57 & 54 \\
Diciembre 2013 & 12 & 14 & 38 & 39 & 494 & 751 & 32 & 36 & 41 & 28 \\
Enero 2014 & 10 & 9 & 36 & 36 & 266 & 249 & 28 & 25 & 27 & 28 \\
Febrero 2014 & 13 & 26 & 36 & 39 & 421 & 1056 & 36 & 67 & 32 & 41 \\
Marzo 2014 & 23 & 28 & 39 & 39 & 1616 & 2009 & 59 & 72 & 70 & 72 \\
Abril 2014 & 16 & 26 & 38 & 34 & 1225 & 2437 & 42 & 76 & 77 & 94 \\
Mayo 2014 & 23 & 25 & 37 & 37 & 1831 & 2285 & 62 & 68 & 80 & 91 \\
Julio 2014 & 14 & 26 & 32 & 34 & 988 & 1063 & 44 & 76 & 71 & 41 \\
Agosto 2014 & 10 & 21 & 34 & 36 & 451 & 779 & 29 & 58 & 45 & 37 \\
Septiembre 2014 & 20 & 19 & 32 & 34 & 1970 & 954 & 63 & 56 & 99 & 50 \\
Octubre 2104 & 18 & 27 & 30 & 32 & 786 & 1112 & 60 & 84 & 44 & 41 \\
Total & 185 & 259 & 429 & 437 & 10927 & 14270 & 522 & 718 & 652 & 633 \\
Prueba-T & $\boldsymbol{p}=\mathbf{0 , 0 0 9}$ & $p=0,873$ & $p=0,3078$ & $\boldsymbol{p}=\mathbf{0 , 0 2 0 0}$ & $p=0,856$ \\
\hline
\end{tabular}

NOP: Número de ovitrampas positivas, NOE: Número de ovitrampas examinadas, NH: Número de huevos, IPO: Índice de positividad de ovitrampas, IDH: índice de huevos.

En cuanto al análisis de correlación entre los índices de oviposición con las variables Precipitación (P) y Temperatura $\left(\mathrm{T}^{\circ}\right)$ se detectó que el área metropolitana se correlacionó en menor medida con las variables de precipitación y temperatura que cuando se comparó individualmente cuando se hizo la comparación con los municipios. En este sentido, el análisis de los municipios evidenció correlaciones positivas $(>0,5)$ en $\mathrm{NOP}, \mathrm{NH}$ e IPO en peridomicilio y IPO y IDH en intradomicilio. De cualquier forma, en Floridablanca fueron evidentes las correlaciones positivas en peridomicilio entre el índice IPO con la precipitación y la temperatura (Tabla 2).
En cuanto al análisis de fluctuación de todas localidades de muestreo, únicamente el municipio de Piedecuesta no presentó oviposición constante en todos los meses (Figuras 2-5). En la ciudad de Bucaramanga la oviposición se evidenció en todos los meses de colecta y fue observado que los índices de ovipisición oscilaron durante el muestro. El índices IDH resultó similar tanto en las colectas intradomicilio como en peridomicilio, sin embargo, se obtuvieron más huevos en el intradomicilio. En este municipio, los mayores picos de oviposición se presentaron en octubre $\mathrm{y}$ mayo-junio para intradomicilio y peridomicilio 
respectivamente para los índices NI y IDH. Los índices más bajos de oviposición fueron en enero para NOP e IPO en peridomicilio (Figura 2).

Tabla 2. Correlaciones de los índices de oviposición obtendidos en el área metropolitana de Bucaramanga.

\begin{tabular}{cccccccccc}
\hline \multirow{2}{*}{ Municipio } & \multirow{2}{*}{$\begin{array}{c}\text { Variable } \\
\text { ambiental }\end{array}$} & \multicolumn{2}{c}{ NOP } & \multicolumn{2}{c}{ IPO } & IDH \\
\cline { 3 - 8 } Intra & Peri & Intra & Peri & Intra & Peri \\
\hline \multirow{2}{*}{ Bucaramanga } & $\mathrm{P}$ & $-0,24$ & 0,16 & $-0,25$ & 0,04 & $-0,34$ & $-0,26$ \\
& $\mathrm{~T}$ & 0,01 & $-0,03$ & 0,27 & 0,13 & 0,18 & $-0,22$ \\
& $\mathrm{P}$ & 0,14 & 0,15 & 0,14 & 0,14 & $-0,11$ & $-0,05$ \\
Floridablanca & $\mathrm{T}$ & 0,27 & 0,48 & 0,37 & 0,6 & 0,42 & 0,39 \\
& $\mathrm{P}$ & 0,01 & 0,63 & 0,23 & 0,75 & $-0,13$ & 0,05 \\
Piedecuesta & $\mathrm{T}$ & 0,3 & 0,54 & 0,55 & 0,66 & 0,04 & 0,21 \\
& $\mathrm{P}$ & $-0,03$ & 0,17 & 0,17 & 0,2 & $-0,01$ & $-0,12$ \\
Área & $\mathrm{T}$ & 0,08 & 0,55 & 0,23 & 0,6 & 0,53 & $-0,36$ \\
metropolitana & $\mathrm{P}$ & $-0,17$ & 0,45 & $-0,19$ & 0,41 & $-0,22$ & 0,05 \\
\hline & $\mathrm{T}$ & 0,48 & $-0,48$ & 0,25 & 0,59 & 0,21 & 0,03 \\
\hline
\end{tabular}

P: precipitación, T: Temperatura, NOP: Número de ovitrampas positivas, NH: Número de huevos, IPO: Índice de positividad de ovitrampas, IDH: índice de huevos
En Girón los índices de oviposición fueron evidentemente mayores en peridomicilio excepto para IDH. En este municipio se presentó un aumento de la oviposición que inició en febrero con su pico en abril con posterior decrecimiento en septiembre (NH y IDH) (Figura 3).

Para el municipio de Floridablanca el índice de oviposición fue mayor en el peridomicilio para IPO. El número de ovitrampas positivas y los índices más altos de oviposición se presentaron en marzo, abril y noviembre (NOP-peridomicilio), noviembre (IPOperidomicilio). Sin embargo, para $\mathrm{NH}$ fue en abril para peridomicilio, pero para IDH se presentó el más alto índice en septiembre para intradomicilio (Figura 4).

En Piedecuesta, los índices en general fueron menores cuando se compararon con los otros municipios. En el mes de enero no se detectó la oviposición de A. aegypti, en septiembre no se detectó oviposicón en septiembre en intradomicilio y mayo-junio se presentó la mayor oviposición para $\mathrm{NH}$, lo cual no fue coincidente con IDH. En este municipio el número de trampas instaladas positivas y los índices de oviposición fueron mayores en intradomicilio para $\mathrm{NH}$ y IDH, sin embargo, para NOP e IPO ocurrió lo contrario (Figura 5).
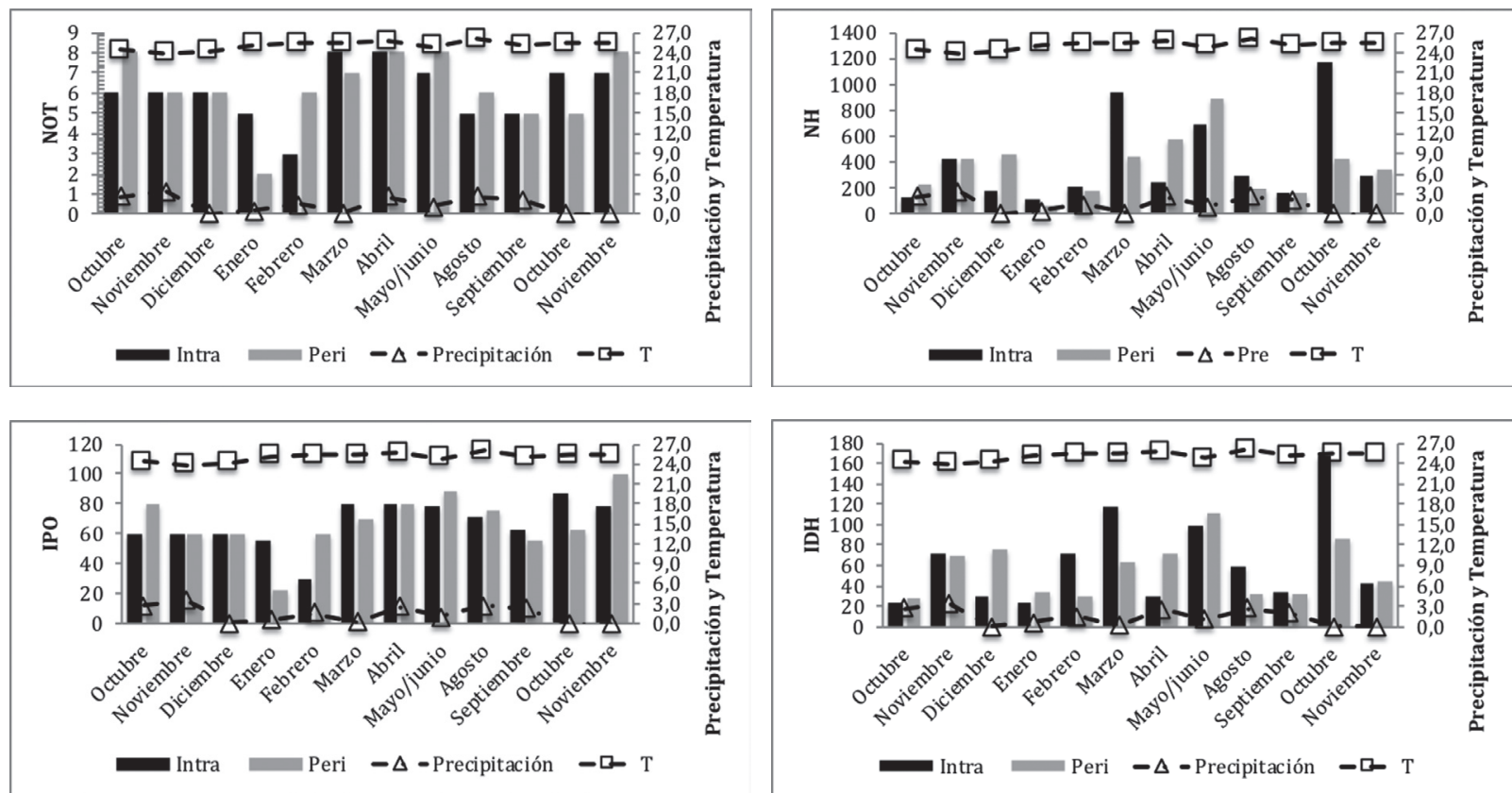

Figura 2. Número de trampas, índices de oviposición y variables climáticas como la presipitación $(\mathrm{P})$ y la temperatura(T) obtendidos en la ciudad de Bucaramanga. 

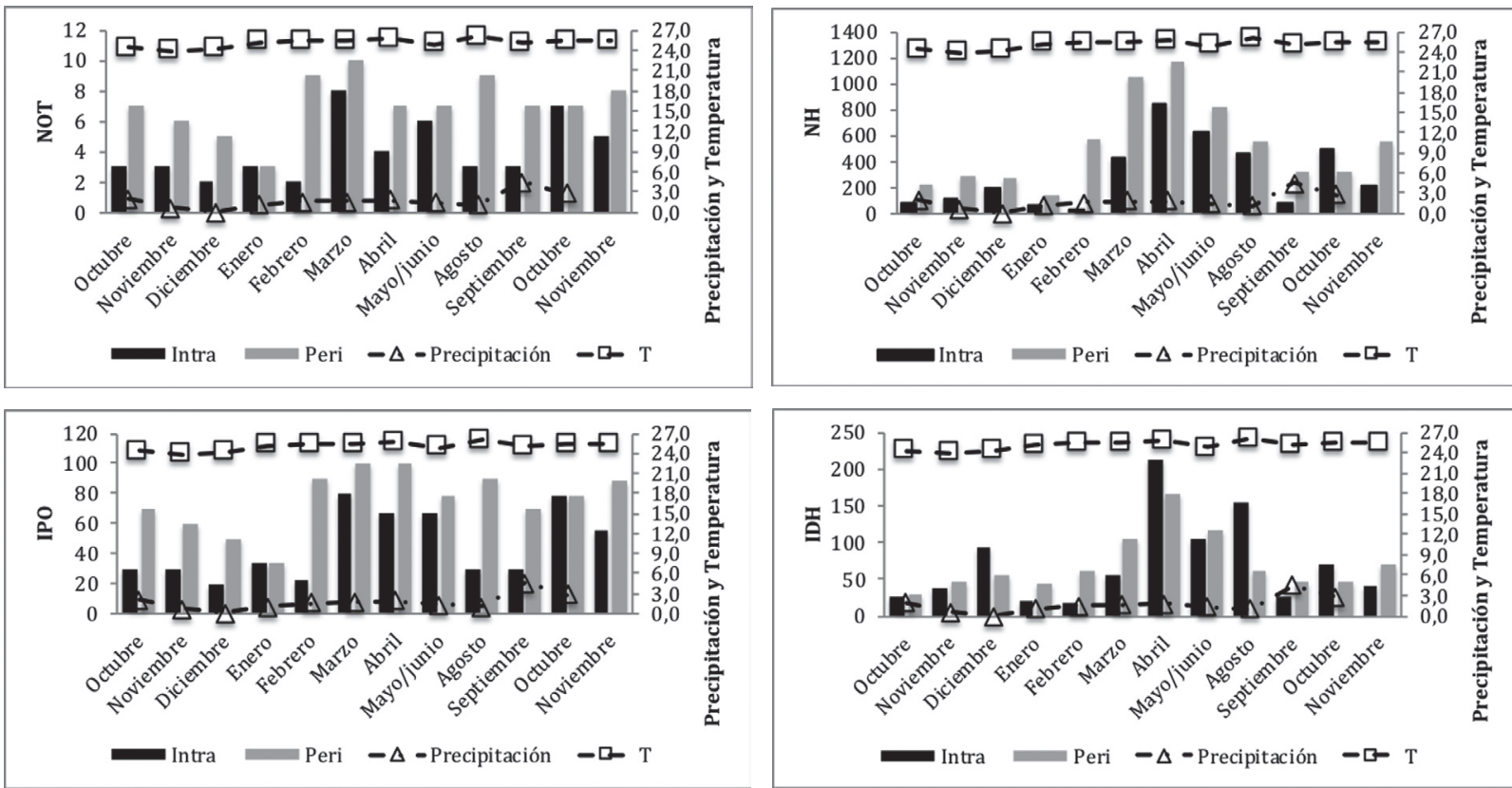

Figura 3. Número de trampas, índices de oviposición y variables climáticas como la presipitación $(\mathrm{P})$ y la temperatura $(\mathrm{T})$ obtendidos en el municipio de Girón.
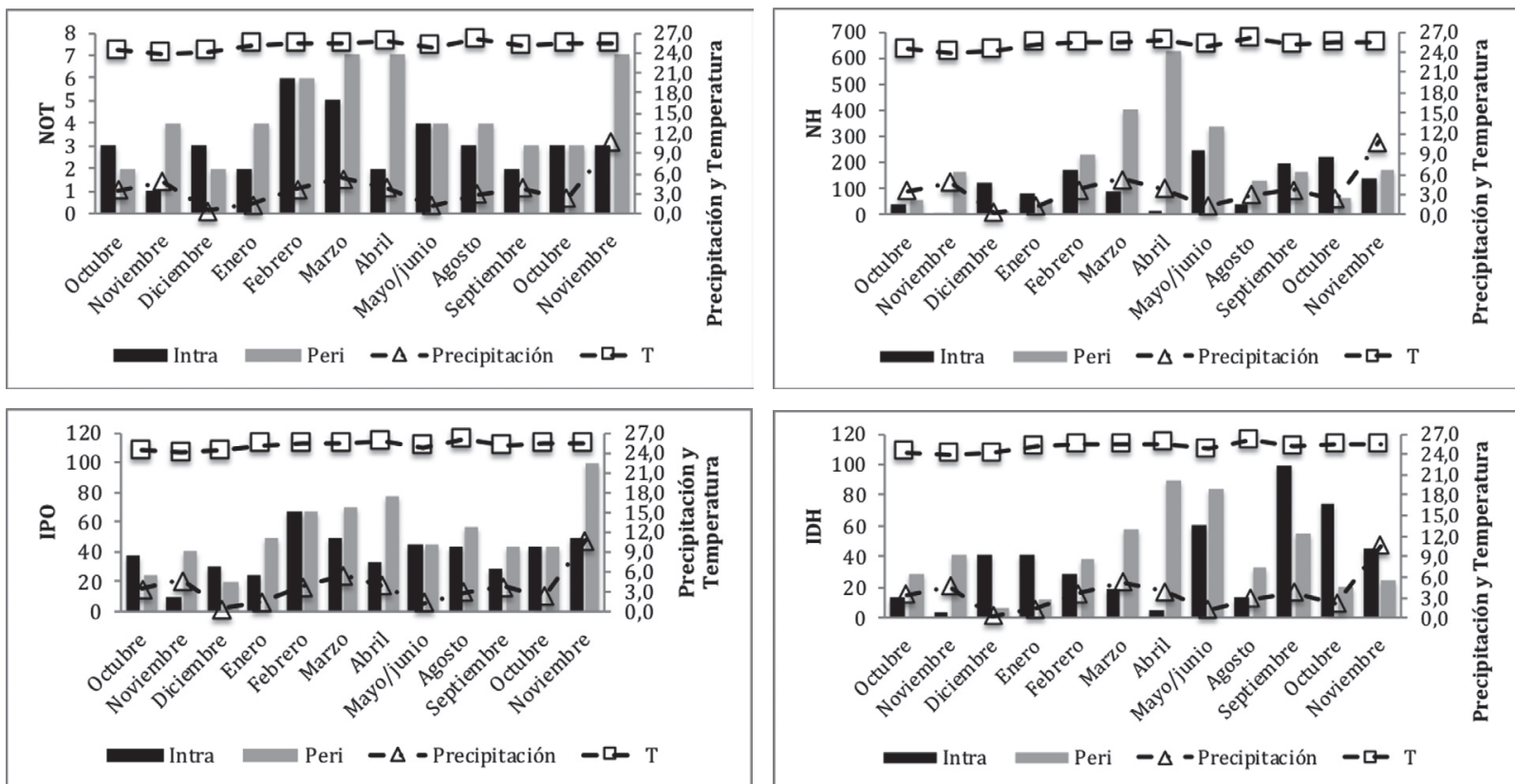

Figura 4. Número de trampas, índices de oviposición y variables climáticas como la presipitación $(\mathrm{P})$ y la temperatura $(\mathrm{T})$ obtendidos en el municipio de Floridablanca.

Se realizó el estado gonotrófico de las hembras colectadas en el área metropolitana en el muestreo activo para un total de 18 hembras de $A$. aegypti y cuatro hembras de Culex quinquefasciatus. Para A. aegypti se determinó que en intradomicilio el $46,7 \%$ de las hembras no presentan actividad hematofágica, 33,3\% presentan un único ciclo gonotrófico y $20 \%$ acusaron dos ciclos gonotróficos. En peridomicilio $66,7 \%$ de las hembras no presentan actividad hematofágica y 33,3\% presentan un único ciclo gonotrófico siendo Girón la localidad con los mosquitos más activos, ya que de seis hembras colectadas cuatro presentaron actividad hematofágica de dos ciclos gonotrófico. Para $C x$. quinquefasciatus se determinó que en el intradomicilio $50 \%$ de las hembras no presentaron actividad hematofágica y el otro $50 \%$ presentó un único ciclo gonotrófico (Tabla 3). 
Durante el muestreo pasivo se colectaron larvas en bromelias, recipientes y llantas de los cuales se encontraron las siguientes especies; Culex spissipes, Culex quinquefasciatus, Limatus durhamii, Toxorhynchites spp y Wyeomyia davisi (Tabla 4).
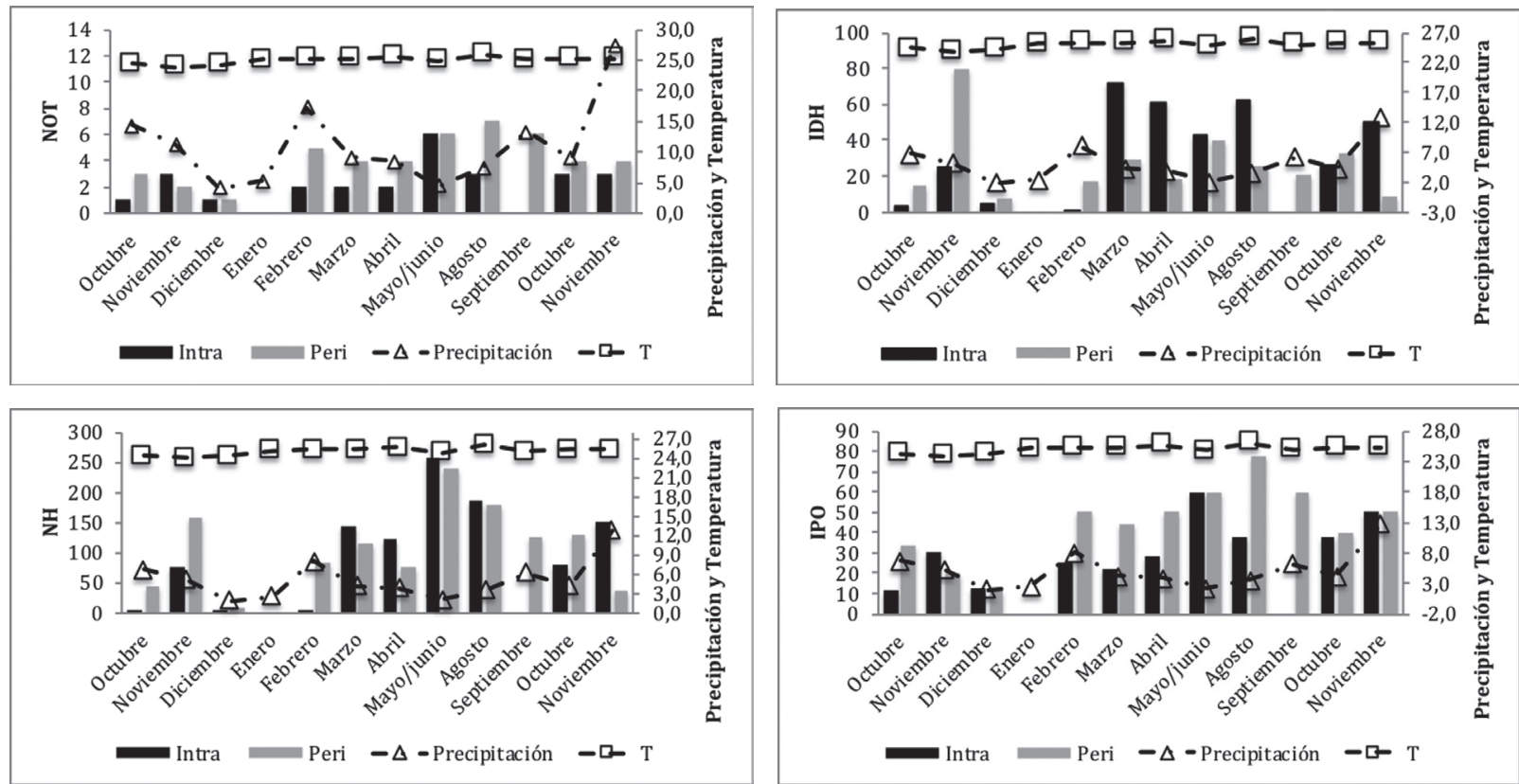

Figura 5. Número de trampas, índices de oviposición y variables climáticas como la presipitación $(\mathrm{P})$ y la temperatura $(\mathrm{T})$ obtendidos en el municipio de Piedecuesta.

Tabla 3. Estados gonadotrófico de mosquitos del área metropolitana de Bucaramanga realizados por aspiración entre mayo y junio.

\begin{tabular}{|c|c|c|c|c|c|c|}
\hline \multirow[b]{2}{*}{ Localidad } & \multirow[b]{2}{*}{ Especie } & \multirow{2}{*}{$\begin{array}{l}\text { Lugar de } \\
\text { captura }\end{array}$} & \multirow{2}{*}{$\begin{array}{c}\text { Total, } \\
\text { analizados* }\end{array}$} & \multicolumn{3}{|c|}{$\%$} \\
\hline & & & & $\begin{array}{c}\text { Ciclo } \\
\text { gonotrófico } 0\end{array}$ & $\begin{array}{c}\text { Ciclo } \\
\text { gonotrófico } 1\end{array}$ & $\begin{array}{c}\text { Ciclo } \\
\text { gonotrófico } 2\end{array}$ \\
\hline \multirow{3}{*}{ Bucaramanga } & A. aegypti & Intra & $2^{+}$ & 100 & 0 & 0 \\
\hline & A. aegypti & Peri & 2 & 50 & 50 & 0 \\
\hline & Cx. quinquefasciatus & Intra & 1 & 100 & 0 & 0 \\
\hline \multirow{2}{*}{ Floridablanca } & A. aegypti & Intra & 1 & 100 & 0 & 0 \\
\hline & Cx. quinquefasciatus & Peri & 1 & 0 & 100 & 0 \\
\hline \multirow{2}{*}{ Giron } & A. aegypti & Intra & 5 & 40 & 0 & 60 \\
\hline & A. aegypti & Peri & 1 & 100 & 0 & 0 \\
\hline \multirow{3}{*}{ Piedecuesta } & A. aegypti & Intra & 7 & 28 & 71 & 0 \\
\hline & A. aegypti & Peri & 1 & 100 & 0 & 0 \\
\hline & Cx. quinquefasciatus & Peri & 2 & 0 & 50 & 0 \\
\hline \multirow{4}{*}{ Área metropolitana } & A. aegypti & Intra & 15 & 46,7 & 33,3 & 20 \\
\hline & A. aegypti & Peri & 3 & 66,7 & 33,3 & 0 \\
\hline & Cx. quinquefasciatus & Intra & 2 & 50 & 50 & 0 \\
\hline & Cx. quinquefasciatus & Peri & 2 & 50 & 50 & 0 \\
\hline
\end{tabular}

* Única colecta en mayo.

${ }^{+}$Un A. aegypti capturado con la trampa StegTrap. 
Tabla 4. Culiciodos capturados en el área metropolitana de Bucaramanga.

\begin{tabular}{|c|c|c|c|c|c|c|}
\hline Localidad & Contenedor & Familia & Género & Especie & Autor & Cantidad \\
\hline \multirow{6}{*}{ Bucaramanga } & \multirow{3}{*}{ Bromelias } & \multirow{3}{*}{ Culicidae } & Toxorhynchites & $\mathrm{sp}$ & (Clark - Gil \& Dorsie, 1983) & 1 \\
\hline & & & Wyeomyia & davisi & (Theobald, 1905) & 6 \\
\hline & & & Aedes & aegypti & (Linnaeus, 1762) & 1 \\
\hline & \multirow{2}{*}{ Aspirado } & \multirow{2}{*}{ Culicidae } & Aedes & aegypti & (Linnaeus, 1762) & 4 \\
\hline & & & Culex & $\mathrm{sp}$ & & 1 \\
\hline & LLanta & Culicidae & Culex & spissipes & (Theobald, 1907) & 1 \\
\hline \multirow{2}{*}{ Floridablanca } & \multirow{2}{*}{ Aspirado } & \multirow{2}{*}{ Culicidae } & Aedes & aegypti & (Linnaeus, 1762) & 1 \\
\hline & & & Culex & spp & & 1 \\
\hline \multirow{6}{*}{ Girón } & \multirow{4}{*}{ Bromelias } & \multirow{2}{*}{ Culicidae } & Wyeomyia & davisi & (Theobald, 1905) & 29 \\
\hline & & & Aedes & aegypti & (Linnaeus, 1762) & 1 \\
\hline & & Psychodidae & & & & 4 \\
\hline & & Sciaridae & & & & 1 \\
\hline & Aspirado & Culicidae & Aedes & aegypti & (Linnaeus, 1762) & 6 \\
\hline & $\begin{array}{l}\text { Recipiente } \\
\text { desechado }\end{array}$ & Culicidae & Culex & quinquefasciatus & (Say, 1826) & 3 \\
\hline \multirow{5}{*}{ Piedecuesta } & \multirow{3}{*}{$\begin{array}{c}\text { Bromelias y } \\
\text { Rutaceas }\end{array}$} & \multirow{3}{*}{ Culicidae } & Limatus & durhamii & Theobald, 1901 & 3 \\
\hline & & & Wyeomyia & davisi & (Theobald, 1905) & 8 \\
\hline & & & Wyeomyia & davisi & (Heobald, 1905) & 8 \\
\hline & \multirow{2}{*}{ Aspirado } & \multirow{2}{*}{ Culicidae } & Aedes & aegypti & (Linnaeus, 1762) & 8 \\
\hline & & & Culex & $\mathrm{sp}$ & & 2 \\
\hline
\end{tabular}

\section{Discusión}

Áreas metropolitanas de los países neotropicales ubicadas por debajo de la altitud de $2200 \mathrm{msnm}$ ofrecen condiciones ideales para la proliferación del zancudo A. aegypti. Sin embargo, esta distribución altitudinal de este insecto se ha ampliado en Colombia hasta los $2302 \mathrm{msnm}^{27}$. Para el caso de este estudio los municipios de Piedecuesta, Floridablanca, y la comuna norte de Bucaramanga además de estar por debajo de la altitud ideal para la proliferación del mosquito ofrecen condiciones de temperatura, humedad y precipitaciones perfectas para que $A$. aegypti se presente de manera constante como se evidenció en esta investigación.

Las ovitrampas resultaron altamente sensitivas para detectar $A$. aegypti como se ha observado en varios países como Brasil, Colombia e India ${ }^{18,28,30-31}$. Se observa cierto grado de coincidencia comparando los resultados estimados de IDH del área metropolitana de Bucaramanga con trabajos similares como el de Romero-Vivas y Falconar $^{31}$ realizado en Puerto Triunfo, departamento del Magdalena. Por ejemplo, el IDH de los dos estudios arrojaron meses similares en cuanto al mayor índice de huevos en los meses de julio, septiembre, y con un bajo índice en noviembre, diciembre y enero. En los trabajos referenciados anteriormente y en estos datos del área metropolitana de Bucaramanga se observa que ninguno se relacionó significativamente con las variables Temperatura y Precipitación lo cuál está acorde con Romero-Vivas y Falconar $^{31}$. En este sentido se detecta que en esta área del trópico el comportamiento de oviposición no sigue un comportamiento estacional como en Argentina Estallo, et al. ${ }^{32}$, Micieli \& Campos $^{33}$ y Codeço, et al. ${ }^{29}$ para el Sur de Brasil. Por el contrario, la presencia de A. aegypti es constante todo el año, lo cual es esperado para esta región del neotrópico.

Sobre el comportamiento de ovoposición de A. aegypti evidenciado por el índice IPO en área peridomiciliar se observó en general mayor que intradomicilio. Este dato podría estar relacionado con una mayor disponibilidad de criaderos en las áreas externas de las viviendas, o podría explicarse también por un posible desplazamiento hacia los exteriores de las viviendas después de alimentarse $\mathrm{e}^{34,35}$. De igual forma, estos datos alertan para tomar medidas de control en el interior de las viviendas pues los índices de oviposición fueron positivos en las dos áreas de muestreo. 
La estimación del ciclo gonadotrófico de A. aegypti y $C x$. quinquefasciatus en las zonas de estudio del área metropolitana de Bucaramanga acusó que un mayor porcentaje de hembras de estas especies no presentaron actividad de oviposición comparado con las que pasaron uno y dos ciclos gonotróficos. Sin embargo, este comportamiento no se repitió en los municipios de Piedecuesta y Giron, en estos lugares, como se detectó para hembras de $A$. aegypti, las cuales evidenciaron en su paridad el paso por uno y dos ciclos gonadotróficos lo cuál concuerda con los reportes para esta especie en Sao Paulo y Rio de Janeiro en Brasil ${ }^{12,36}$. De cualquier forma, estos resultados aunque no son nuevos para estas especies, si evidencian una aproximación de lo que puede estar pasando en el área metropolitana de Bucaramanga sobre la discordancia gonadotrofica de estos zancudos, lo cuál en su comportamiento indica que realiza varias alimentaciones hasta completar la cantidad de sangre adecuada para madurar sus huevos y ovipositar ${ }^{26,37-40}$.

En cuanto a las especies de mosquitos recolectadas en este estudio, se observó que además de $A$. aegypti están presentes otros mosquitos de importancia médica como Cx. quinquefasciatus, Cx. spissipes, Wyeomyia davisi y Limatus durhamii ${ }^{21-23}$. Báscicamente, la presencia de estas especies fue posible por los diferentes criaderos de culicidae y la abundante vegetación ornamental dispuesta que estaban en los lugares de colecta. Sin embargo, de forma inusual y en menor cantidad que las otras especies de zancudos en este estudio se detectó la presencia de $A$. aegypti en bromelias en las localidades de Bucaramanga, Floridablanca y Giron. En este sentido, se observa al igual que en otros estudios como A. aegypti puede estar presente en criaderos diferentes a los artificiales ${ }^{40-42}$. Las colectas activas permitieron evidenciar que $A$. aegypti compartió las bromelias como criadero con Wyeomyia davisi y Toxorhynchites spp. Sin embargo, esta especie generalmente se encuentra compartiendo hábitat con A. albopictus con más frecuencia que otros culícidos en criaderos naturales y artificiales ${ }^{16,43-44}$.

Si bien, otro aspecto para resaltar es que en las bromelias se detectó tambien Toxorhynchites spp, mosquito que no presenta habitos hematofagos y que ejerse control de larvas por su hábitos depredadores de los géneros Wyeomyia y Limatus colectados en criaderos naturales como las bromelias, bambus y huecos de arboles ${ }^{45,46}$.

También llama la atención con este estudio la presencia de Culex quinquefasciatus en el área metropolitana de Bucaramanga, este zancudo, generalmente tiene su nicho en lugares donde la disponibilidad de criaderos se relacionan con aguas altamente eutroficadas, a pesar de esto, se detectó dentro de las viviendas, lo cuál resulta en una alerta pues se ha especulado que esta especie también puede transmitir Zika ${ }^{47}$.

En conclusión, para este primer estudio entomológico realizado en el área metropolitana de Bucaramanga, fueron detectadas diferentes especies de zancudos, siendo Aedes aegypti la especie presente en todo el año.

\section{Agradecimientos}

A la Vicerrectoría de Investigación de la Universidad industrial de Santander por financiar los proyectos de investigación 1362 y 5696. A las secretarias de Salud de Bucaramanga, Girón, Floridablanca por las recomendaciones en los lugares de colecta. A las familias de los barrios Divino Niño comuna Norte de Bucaramanga, Lagos dos de Floridablanca, al conjunto Alicante en Girón y Hacienda San Miguel de Piedecuesta y al Dr. Eduardo Fumio Kuwabara por la revisión y confirmación de las especies de zancudos reportadas en este estudio.

\section{Conflicto de interés}

Los autores de este artículo declaran no tener conflictos de interés

\section{Referencias}

1. Rozo-Lopez P, Mengual X. Updated list of the mosquitoes of Colombia (Diptera: Culicidae). Biodivers Data J. 2015; 3: e4567. doi: 10.3897/ BDJ.3.e4567.

2. Thiberville SD, Moyen N, Dupuis-Maguiraga L, Nougairede A, Gould EA, Roques P, et al. Chikungunya fever: Epidemiology, clinical syndrome, pathogenesis and therapy. Antiviral Res. 2013; 99(3): 345-370. doi: 10.1016/j. antiviral.2013.06.009.

3. Guzman MG, Halstead SB, Artsob H, Buchy P, Farrar J, Gubler DJ, et al. Dengue : a continuing global threat. Nat Rev Microbiol. 2010; 8(12Suppl): S7-16. doi: 10.1038/nrmicro2460.

4. Caglioti C, Lalle E, Castilletti C, Carletti F, Capobianchi MR, Bordi L. Chikungunya virus infection: an overview. New Microbiol. 2013; 36(3): 211-227.

5. Duque JE, Navarro-Silva M, Trejos D. Simulando manejo de Aedes aegypti (Diptera: Culicidae) y sus efectos en una epidemia de dengue. Rev Col Entomol. 2009; 35(1): 66-72. 
6. Olano VA, Matiz MI, Lenhart A, Cabezas L, Vargas SL, Jaramillo JF, et al. Schools as Potential Risk Sites for Vector-Borne Disease Transmission: Mosquito Vectors in Rural Schools in Two Municipalities in Colombia. J Am Mosq Control Assoc. 2015; 31(3): 212-222. doi: 10.2987/moco-31-03-212-222.1.

7. Méndez F, Barreto M, Arias JF, Rengifo G, Muñoz J, Burbano ME, et al. Human and mosquito infections by dengue viruses during and after epidemics in a dengue-endemic region of Colombia. Am J Trop Med Hyg. 2006; 74(4): 678-683.

8. Villar LÁ. Dengue: un reto para el estado, la comunidad científica y el conjunto de la sociedad colombiana. Infect. 2011; 15(1): 5-7.

9. Gubler DJ. Dengue and Dengue Hemorrhagic Fever. 1998;11(3): 480-496.

10. Achee NL, Gould F, Perkins TA, Reiner RC, Morrison AC, Ritchie SA, et al. A Critical Assessment of vector control for dengue prevention. PLoS Negl Trop Dis. 2015; 9(5): e0003655. doi: 10.1371/journal.pntd.0003655.

11. Goindin D, Delannay C, Ramdini C, Gustave J, Fouque F. Parity and longevity of Aedes aegypti according to temperatures in controlled conditions and consequences on dengue transmission risks. PLoS One. 2015; 10(8): 1-21. doi: 10.1371/journal. pone.0135489.

12. Lima-Camara TN, Honório NA, Lourenço-deOliveira R. Parity and ovarian development of Aedes aegypti and Ae. albopictus (Diptera: Culicidae) in metropolitan Rio de Janeiro. J Vector Ecol. 2007; 32(1): 34-40. doi: https://doi.org/10.3376/10811710(2007)32[34:PAODOA]2.0.CO;2.

13. Ocazionez-Jiménez R, Ortiz-Báez AS, GómezRangel SY, Miranda-Esquivel DR. Virus del dengue de serotipo 1 (DENV-1) de Colombia: su contribución a la presentación del dengue en el departamento de Santander. Biomédica. 2013; 33: 22-30. doi: http://dx.doi.org/10.7705/biomedica. v33i0.717.

14. Cáceres-Manrique FM, Angulo-Silva ML, Vesga-Gómez C. Eficacia de la movilización y la participación social para la apropiación o "empoderamiento" (sic.) (empowerment) de las medidas de control del dengue, Comuna Norte, Bucaramanga, 2008-2009. Biomedica. 2010; 30(4): 539-550.

15. Reiter P. Oviposition, dispersal, and survival in Aedes aegypti: implications for the efficacy of control strategies. Vector-Borne Zoonotic Dis. 2007;7(2): 261-273. doi: 10.1089/vbz.2006.0630.

16. Fantinatti EC, Duque JEL, Silva A, Navarro-Silva M. Abundância e Agregação de Ovos de Aedes aegypti L. Aedes albopictus (Skuse) (Diptera: Culicidae ) no Norte e Noroeste do Paraná. Neotrop Entomol. 2007; 36(6): 960-965. doi: http://dx.doi. org/10.1590/S1519-566X2007000600020.

17. de Resende MC, de Ázara TMF, Costa IO, Heringer LC, de Andrade MR, Acebal JL, et al. Field optimisation of mosquiTRAP sampling for monitoring Aedes aegypti Linnaeus (Diptera: Culicidae). Mem Inst Oswaldo Cruz. 2012; 107(3): 294-302. doi: http://dx.doi.org/10.1590/S007402762012000300002.

18. Honório NA, Codeço CT, Alves FC, Magalhães MAFM, Lourenço-de-Oliveira R. Temporal distribution of Aedes aegypti in different districts of Rio de Janeiro, Brazil, measured by two types of traps. J Med Entomol. 2009; 46(5): 1001-1014.

19. Gomes ADC. Vigilância Entomológica. Inf Epidemiológico do Sist Único Saúde. 2002; 11(2): 79-90.

20. Miyazaki RD, Ribeiro ALM, Pignatti MG, Júnior JHC, Pignati M. Monitoramento do mosquito Aedes aegypti (Linnaeus , 1762) (Diptera: Culicidae), por meio de ovitrampas no Campus da Universidade Federal de Mato Grosso, Cuiabá, Estado de Mato Grosso. Rev Soc Bras Med Trop. 2009; 42(4): 392-397. doi: http://dx.doi.org/10.1590/S003786822009000400007.

21. Forttini O. Identificação, biologia e epidemiologia. Culicidologia Médica. V2 ed. Universidade de Sao Paulo. 2002; 851 p.

22. Belkin J, Schick R, Heinemann SJ. Contributions of American the Entomological Institute. Vol. 7, Mosquito studies. 1971; 1-67 p.

23. Cova Garcia P, Sutil E, Rausseo JA. Mosquitos (Culicinos) de Venezuela. 1966; 245 p.

24. Torres JP, Aranda MN, Moreno HJP, Duque JE. Design and construction of a trap for the surveillance of Aedes (Stegomyia) aegypti. Rev Univ Ind Santander. 2016; 48(3): 285-294. doi: http://dx.doi. org/10.18273/revsal.v48n3-2016002.

25. Macdonald G, Detinova T, Bertram D. Course in advanced entomological techniques applied to malaria eradication. London; 1959.

26. Charlwood J, Rafael J, Wilkes T. Métodos de determinar a idade fisiológica em Diptera de importância médica. Uma revisão com especial referência aos vetores de doenças na América do Sul. Acta Amaz. 1980; 10(2): 311-333. doi: http:// dx.doi.org/10.1590/1809-43921980102311.

27. Ruiz-López F, González-Mazo A, Vélez-Mira A, Gómez GF, Zuleta L, Uribe S, et al. Presencia de Aedes (Stegomyia) aegypti (Linnaeus, 1762) $\mathrm{y}$ su infección natural con el virus del dengue en 
alturas no registradas para Colombia. Biomédica. 2016; 36(2): 303-308. doi: https://doi.org/10.7705/ biomedica.v36i2.3301.

28. Alarcón ÉP, Segura ÁM, Rúa-Uribe G, Parra-Henao G. Evaluación de ovitrampas para vigilancia y control de Aedes aegypti en dos centros urbanos del Urabá antioqueño. Biomédica. 2014; 34: 409-424. doi: https://doi.org/10.7705/biomedica.v34i3.2134.

29. Codeço CT, Lima AWS, Araújo SC, Lima JBP, Maciel-de-Freitas R, Honório NA, et al. Surveillance of Aedes aegypti: Comparison of House Index with Four Alternative Traps. PLoS Negl Trop Dis. 2015; 9(2): 1-23. doi: 10.1371/journal.pntd.0003475.

30. Sivagnaname N, Gunasekaran K. Need for an efficient adult trap for the surveillance of dengue vectors. Indian J Med Res. 2012;136: 739-749.

31. Romero-Vivas CME, Falconar AKI. Investigation of relationships between Aedes aegypti egg, larvae, pupae, and adult density indices where their main breeding sites were located indoors. J Am Mosq Control Assoc. 2005; 21(1): 15-21. doi: 10.2987/8756-971X(2005)21[15:IORBAA]2 .0. $\mathrm{CO} ; 2$.

32. Estallo EL, Ludueña-Almeida FF, Introini M V, Zaidenberg M, Almirón WR. Weather variability associated with Aedes (Stegomyia) aegypti (Dengue Vector) oviposition dynamics in Northwestern Argentina. PLoS One. 2015; 10(5): 1-11. doi: 10.1371/journal.pone.0127820.

33. Micieli MV, Campos RE. Oviposition Activity and Seasonal Pattern of a Population of Aedes (Stegomyia) aegypti (L.) (Diptera: Culicidae) in Subtropical Argentina. Mem Inst Oswaldo Cruz. 2003; 98(5): 659-663.

34. Casas-Martínez M, Orozco-Bonilla A, MuñozReyes M, Ulloa-García A, Bond JG, Valle-Mora J, et al. A new tent trap for monitoring the daily activity of Aedes aegypti and Aedes albopictus. J Vector Ecol. 2013; 38(2): 277-288. doi: 10.1111/j.19487134.2013.12041.x.

35. Chadee DD, Martinez R. Landing periodicity of Aedes aegypti with implications for dengue transmission in Trinidad, West Indies. J vector Ecol. 2000; 25(2): 158-163.

36. Fávaro EA, Dibo MR, Mondini A, Ferreira AC, Barbosa AAC, Eiras ÁE, et al. Physiological state of Aedes (Stegomyia) aegypti mosquitoes captured with MosquiTRAPs in Mirassol, São Paulo, Brazil. J Vector Ecol. 2006; 31(2): 285-291.

37. Maciel-De-Freitas R. A review on the ecological determinants of Aedes aegypti (Diptera: Culicidae) Vectorial Capacity. Oecologia Austral. 2010; 14(3): 726-736. doi: 10.4257/oeco.2010.1403.08.
38. Ball TS, Ritchie SR. Sampling Biases of the BGSentinel Trap With Respect to Physiology, Age, and Body Size of Adult Aedes aegypti (Diptera: Culicidae). J Med Entomol. 2010; 47(4): 649-656.

39. Barata EAMF, Costa AIP, Chiaravalloti-Neto F, Barata JMS, Natal D. População de Aedes aegypti (1.) em área endêmica de dengue, Sudeste do Brasil. Rev Saude Publica. 2001; 35(3): 237-242. doi: http:// dx.doi.org/10.1590/S0034-89102001000300004.

40. Forattini OP, Marques GRAM. Nota sobre o encontro de Aedes aegypti em bromélias. Rev Saude Publica. 2000; 34(5): 543-544. doi: http://dx.doi. org/10.1590/S0034-89102000000500016.

41. Cunha SP, Alves JRC, Lima MM, Duarte JR, Barros LC De, Silva JL Da, et al. Presença de Aedes aegypti em Bromeliaceae e depósitos com plantas no Município do Rio de Janeiro, RJ. Rev Saude Publica. 2002; 36(2): 244-245. doi: http://dx.doi. org/10.1590/S0034-89102002000200018.

42. Oliveira VC de, Almeida Neto LC de. Ocorrência de Aedes aegypti e Aedes albopictus em bromélias cultivadas no Jardim Botânico Municipal de Bauru, São Paulo, Brasil. Cad Saude Publica. 2017; 33(1): 1-7. doi: http://dx.doi.org/10.1590/0102$311 \times 00071016$.

43. Calderón O, Troyo A, Solano M. Diversidad larval de mosquitos (Diptera: Culicidae) en contenedores artificiales procedentes de una comunidad urbana de San José, Costa Rica. Parasitol Latinoam. 2004; 59(3-4): 132-136.

44. Rodríguez RM, Ríos MD, Álvarez Gutiérrez Y, Calderón Arguedas O. Sitios de cria de Aedes aegypti (Linnaeus) y distribución geográfica de Aedes albopictus (Skuse) en la provincia de Limón, Costa Rica. Rev Cubana Med Trop. 2014; 66(2): 219-227.

45. Liria J. Fauna fitotelmata en las bromelias Aechmea fendleri André y Hohenbergia stellata Schult del Parque Nacional San Esteban, Venezuela. Rev Peru Biol. 2007; 14(1): 33-38.

46. Barajas J, Suaza J, Torres C, Rua G, Uribe S, Porter C. Mosquitos (Diptera: Culicidae) asociados a guadua en los municipios de Anserma, Hispania y Jardín, Colombia. Rev Colomb Entomol. 2013; 39(1): 132-140.

47. Guedes DR, Paiva MH, Donato MM, Barbosa PP, Krokovsky L, Rocha SW dos S, et al. Zika virus replication in the mosquito Culex quinquefasciatus in Brazil. Emerg Microbes Infect. 2017; 6(8): e69. doi: 10.1038/emi.2017.59. 